\title{
Amphibians of Santa Teresa, Brazil: the hotspot further evaluated
}

\author{
Rodrigo Barbosa Ferreira' ${ }^{1,2}$, Alexander Tamanini Mônico ${ }^{1,3}$, \\ Emanuel Teixeira da Silva ${ }^{4,5}$, Fernanda Cristina Ferreira Lirio', Cássio Zocca',3, \\ Marcio Marques Mageski', João Filipe Riva Tonini6,7, Karen H. Beard ${ }^{2}$, \\ Charles Duca', Thiago Silva-Soares ${ }^{3}$
}

I Programa de Pós-Graduação em Ecologia de Ecossistemas, Universidade Vila Velha, Campus Boa Vista, 29102-920, Vila Velha, ES, Brazil 2 Department of Wildland Resources and the Ecology Center, Utah State University, Logan, UT, USA 3 Instituto Nacional da Mata Atlântica/Museu de Biologia Prof. Mello Leitão, 29650-000, Santa Teresa, ES, Brazil 4 Laboratório de Herpetologia, Departamento de Zoologia, Instituto de Ciências Biológicas, Universidade Federal de Minas Gerais, Avenida Antônio Carlos, 6627, Pampulha, Belo Horizonte, MG, Brazil 5 Centro de Estudos em Biologia, Centro Universitário de Caratinga, Avenida Niterói, s/n, Bairro Nossa Senhora das Graças, 35300-000, Caratinga, MG, Brazil 6 Department of Organismic and Evolutionary Biology, Harvard University, 26 Oxford St, Cambridge, MA, USA 7 Museum of Comparative Zoology, Harvard University, 26 Oxford St, Cambridge, MA, USA

Corresponding author: Rodrigo Barbosa Ferreira (rodrigoecologia@yahoo.com.br)

Academic editor: A. Crottini | Received 4 October 2018 | Accepted 20 April 2019 | Published 25 June 2019

http://zoobank.org/1923497F-457B-43BA-A852-5B58BEB42CC1

Citation: Ferreira RB, Mônico AT, da Silva ET, Lirio FCF, Zocca C, Mageski MM, Tonini JFR, Beard KH, Duca C, Silva-Soares T (2019) Amphibians of Santa Teresa, Brazil: the hotspot further evaluated. ZooKeys 857: 139-162. https://doi.org/10.3897/zookeys.857.30302

\begin{abstract}
A checklist of the amphibians of Santa Teresa municipality, in southeastern Brazil is presented based on fieldwork, examination of specimens in collections, and a literature review. This new amphibian list of Santa Teresa includes 108 species, of which $106(-98 \%)$ belong to Anura and two $(-2 \%)$ to Gymnophiona. Hylidae was the most represented family with 47 species (43\%). Compared to the previous amphibian lists for Santa Teresa, 14 species were added, 17 previously reported species were removed, and 13 species were re-identified based on recent taxonomic rearrangements. Of the 14 species added, 11 (79\%) were first recorded during our fieldwork and specimen examination. It is also the first list of caecilians for Santa Teresa. This list suggests that Santa Teresa has 0.16 species per $\mathrm{km}^{2}$ (i.e., 108 species $/ 683 \mathrm{~km}^{2}$ ), one of the highest densities of amphibian species in the world at a regional scale. This richness represents $78 \%$ of the
\end{abstract}

Copyright Rodrigo Barbosa Ferreira et al. This is an open access article distributed under the terms of the Creative Commons Attribution License (CC BY 4.0), which permits unrestricted use, distribution, and reproduction in any medium, provided the original author and source are credited. 
136 anurans from Espírito Santo state and 10\% of the 1,080 amphibians from Brazil. We highlight the need for long-term monitoring to understand population trends and develop effective conservation plans to safeguard this remarkable amphibian richness.

\section{Keywords}

Anura, Atlantic Forest, Caecilians, Diversity, Espírito Santo, Inventory

\section{Introduction}

Species checklists provide a scientific value to areas by identifying the richness that is threatened given anthropogenic actions. The Brazilian Amphibian Conservation Action plan recognizes that species lists are a scientific priority for many areas across Brazil (Verdade et al. 2012). For instance, Brazil's Atlantic Forest is one of the most threatened global biodiversity hotspots and remains under-sampled given the high number of new species recently described (Lourenço-de-Moraes et al. 2014, Ferreira et al. 2015, Marciano-Jr et al. 2017). The Atlantic Forest has currently $12 \%$ of its historical range, which has resulted in the replacement of continuous forest to small remnants surrounded by human settlements, pastures, plantations, and roads (Ribeiro et al. 2009, Tabarelli et al. 2010). Thus, compiling data regarding the biodiversity of this tropical forest is a conservation priority, especially because several studies have detected changes and declines of some species (Heyer et al. 1988, Weygoldt 1989, Carvalho et al. 2017).

The Atlantic Forest harbors 625 anuran species and 14 caecilians (Rossa-Feres et al. 2017). The state of Espírito Santo, southeastern Brazil harbors 136 (22\%) species listed for Atlantic Forest. The state's most sampled area is the municipality of Santa Teresa, which comprises high functional and phylogenetic diversity of amphibians (Almeida et al. 2011, Campos et al. 2017, Lourenço-de-Moraes et al. 2019). There are conflicting reports regarding the species composition and richness in this area. The first species list for Santa Teresa recorded 102 anuran species (Rödder et al. 2007). However, the state list of anurans mentioned 92 species for Santa Teresa (Almeida et al. 2011). In recent years, new species have been described for Santa Teresa (e.g., Lourenço-de-Moraes et al. 2014, Ferreira et al. 2015, Taucce et al. 2018), some species have been reported for the first time in the area (Simon and Peres 2012), and there have been many taxonomic changes (e.g., Pimenta et al. 2014, Walker et al. 2016), indicating the need to update the species list of this anuran diversity hotspot.

Santa Teresa is also a hotspot for several other taxa, such as plants (Thomaz and Monteiro 1997), birds (Simon 2000), butterflies (Brown and Freitas 2000), and small mammals (Passamani et al. 2000). Due to its remarkable biological importance, it is essential to keep the species lists updated. Here, we present an updated species list of the amphibians for Santa Teresa based on many years of fieldwork, examination of specimens from scientific collections, and literature review. 


\section{Materials and methods}

\section{Study area}

The municipality of Santa Teresa has $683 \mathrm{~km}^{2}$ and is located in the mountainous region (altitude range: -120-1099 m a.s.l.) of Espírito Santo state, southeastern Brazil $\left(19^{\circ} 56^{\prime} 14\right.$ "S, $40^{\circ} 35^{\prime} 52^{\prime \prime W}$; Figure 1). Santa Teresa encompasses the southern portion of Bahia Coastal Forests ecoregion, and northern portion of Serra do Mar ecoregion in the Atlantic Forest (Olson et al. 2001, Scaramuzza et al. 2011, Campos and Lourençode-Moraes 2017, Silva et al. 2018).

The predominant vegetation types are montane and sub-montane rainforests (Rizzini 1979), characterized by non-deciduous trees with lead buds without protection against drought (Brasil 1983). Santa Teresa was mostly forested until the arrival of European settlers in 1874 . Currently, the municipality has $42 \%$ of its original forest cover inside and surrounding three protected areas: the Reserva Biológica Augusto Ruschi (3,598 ha), the Estação Biológica de Santa Lúcia (440 ha), and the Parque Natural de São Lourenço (22 ha) (SOS Mata Atlântica and Inpe 2013). Outside these protected areas, forest remnants are in private properties and mostly restricted to hilltops while the valleys are dominated by different types of human-modified matrix (e.g., coffee plantations, Eucalyptus spp. plantations, abandoned pastures, and settlements; Ferreira et al. 2016).

The climate of Santa Teresa is classified as oceanic climate without dry season and with temperate summer (Cfb) according to Köppen classification (Alvares et al. 2013). Mean annual precipitation is $1,868 \mathrm{~mm}$ with highest rainfall in November and lowest in June, when the mean rainfall is less than $60 \mathrm{~mm}$ (Mendes and Padovan 2000). Mean annual temperature is $20^{\circ} \mathrm{C}$ (range: $14.3-26.2^{\circ} \mathrm{C}$, Thomaz and Monteiro 1997).

\section{Data sampling}

The species list presented in this study has been compiled in part using field surveys conducted by the authors from 2006 to 2019, and also through the evaluation of specimens in zoological collections (see Appendix I) and a literature review.

During field surveys, we conducted intensive sampling across Santa Teresa using audio and visual searches inside bromeliads, in the leaf litter, and in water bodies (see Dodd 2010). We released easily identified and extensively vouchered (> 30 specimens) species but took those species with more complex identification back to laboratory. To do this, we kept amphibians in moist plastic tubes or plastic bags to prevent dehydration. Some specimens were euthanized by ventral application of $7.5 \%$ to $20 \%$ benzocaine, preserved using 10\% formalin and then transferred to 70\% ethanol (American and Veterinary Medical Association 2013, CEBEA/CFMV 2013).

We also reviewed the literature and compiled records of amphibians for Santa Teresa. In addition, we examined specimens deposited in the following institutions: 


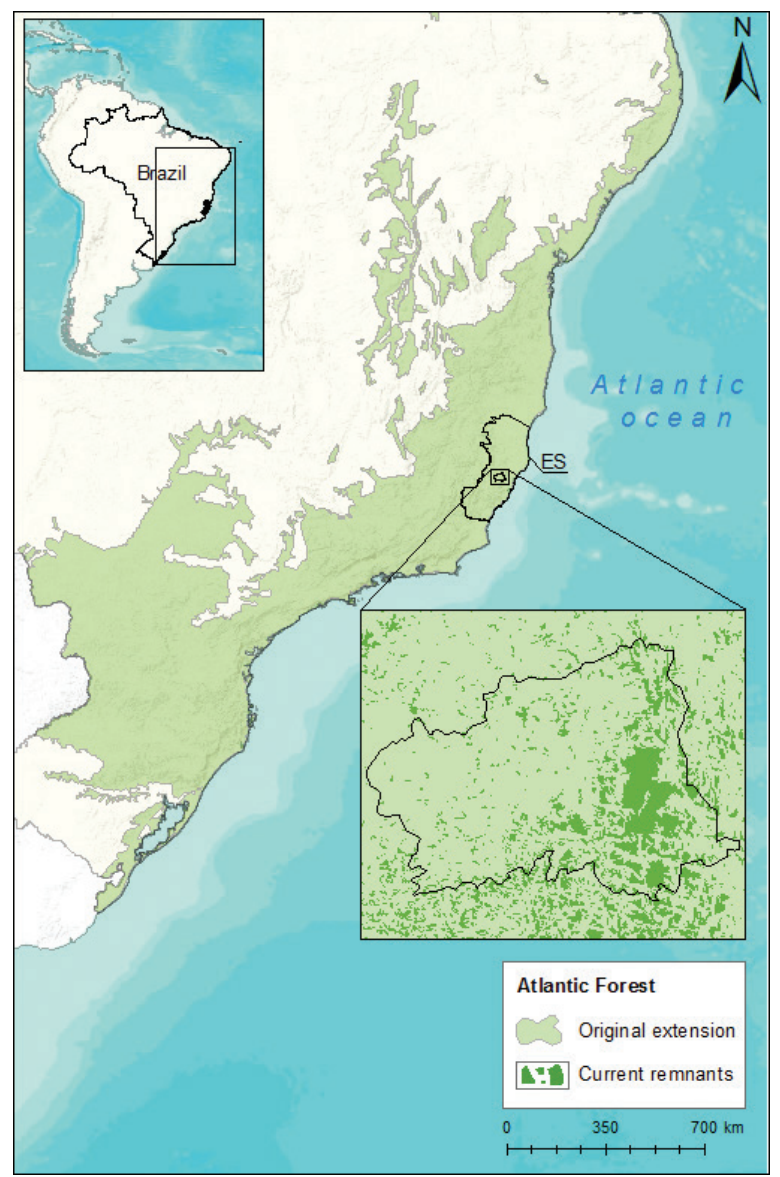

Figure I. Location of the municipality of Santa Teresa, southeastern Brazil. Forest remnants from SOS Mata Atlântica (2014).

Coleção de Anfíbios Célio F. B. Haddad (CFBH), Universidade Estadual Paulista (UNESP); Museu de Biologia Mello Leitão (MBML), Instituto Nacional da Mata Atlântica (INMA); Universidade Federal de Minas Gerais (UFMG); Museu Nacional, Universidade Federal do Rio de Janeiro (MNRJ); Museu de Zoologia Prof. Adão José Cardoso (ZUEC), Universidade Estadual de Campinas (UNICAMP); Museu de Zoologia, Universidade de São Paulo (MZUSP); and Smithsonian National Museum of Natural History (USNM) (see Appendix I). We followed Frost (2019) for taxonomic arrangements.

\section{Results}

We recorded 108 amphibian species for Santa Teresa, of which 106 (98\%) belong to Anura (16 families and 41 genera) and two (2\%) to Gymnophiona (one family and one genus) (Table 1; Figure 2, 3, 4, 5, 6, 7). The most represented families were Hylidae 
Table I. Amphibian species of Santa Teresa municipality, Espírito Santo state, Southeastern Brazil. An asterisk * indicates a taxonomic change.

\begin{tabular}{|c|c|c|c|c|}
\hline Species by Family & $\begin{array}{c}\text { Type } \\
\text { locality }\end{array}$ & $\begin{array}{c}\text { Our } \\
\text { study }\end{array}$ & $\begin{array}{c}\text { Almeida } \\
\text { et al. } 2011\end{array}$ & $\begin{array}{c}\text { Rödder } \\
\text { et al. } 2007\end{array}$ \\
\hline \multicolumn{5}{|l|}{ AROMOBATIDAE } \\
\hline Allobates capixaba (Lutz, 1925) & & $\mathrm{X}$ & $\mathrm{X}$ & $\mathrm{X}^{*}$ \\
\hline \multicolumn{5}{|l|}{ BRACHYCEPHALIDAE } \\
\hline Brachycephalus alipioi Pombal \& Gasparini, 2006 & & $\mathrm{X}$ & $\mathrm{X}$ & - \\
\hline Brachycephalus aff. didactylus & & $\mathrm{X}$ & - & - \\
\hline Ischnocnema abdita Canedo \& Pimenta, 2010 & $\mathrm{X}$ & $\mathrm{x}$ & $\mathrm{X}$ & - \\
\hline Ischnocnema colibri Taucce, Canedo, Parreiras, Drummond, Nogueira- & $\mathrm{X}$ & $\mathrm{X}$ & - & - \\
\hline \multicolumn{5}{|l|}{ Costa \& Haddad, 2018} \\
\hline Ischnocnema epipeda (Heyer, 1984) & $\mathrm{X}$ & $\mathrm{X}$ & $\mathrm{X}$ & $\mathrm{X}$ \\
\hline Ischnocnema aff. guentheri & & $\mathrm{X}$ & $\mathrm{X}^{*}$ & $\mathrm{X}^{*}$ \\
\hline Ischnocnema cf. nasuta (Lutz, 1925) & & $\mathrm{X}$ & $\mathrm{X}^{*}$ & $\mathrm{X}^{*}$ \\
\hline Ischnocnema oea (Heyer, 1984) & $\mathrm{X}$ & $\mathrm{X}$ & $\mathrm{X}$ & $\mathrm{X}$ \\
\hline Ischnocnema aff. parva sp. 1 & & $\mathrm{X}$ & $\mathrm{X}^{*}$ & $\mathrm{X}^{*}$ \\
\hline Ischnocnema aff. parva sp. 2 & & $\mathrm{X}$ & - & - \\
\hline Ischnocnema verrucosa Reinhardt \& Lütken, 1862 & & $\mathrm{X}$ & $\mathrm{X}$ & $\mathrm{X}$ \\
\hline \multicolumn{5}{|l|}{ BUFONIDAE } \\
\hline Dendrophryniscus carvalhoi Izecksohn, 1994 & $\mathrm{X}$ & $\mathrm{X}$ & $\mathrm{X}$ & $\mathrm{X}$ \\
\hline Rhinella crucifer (Wied-Neuwied, 1821) & & $\mathrm{X}$ & $\mathrm{X}$ & $\mathrm{X}$ \\
\hline Rhinella granulosa (Spix, 1824) & & $\mathrm{X}$ & $\mathrm{X}$ & $\mathrm{X}$ \\
\hline Rhinella diptycha (Cope, 1862) & & $\mathrm{X}$ & $\mathrm{X}$ & $\mathrm{X}$ \\
\hline \multicolumn{5}{|l|}{ CENTROLENIDAE } \\
\hline Vitreorana aff. eurygnatha & & $\mathrm{X}$ & $\mathrm{X}^{*}$ & $\mathrm{X}^{*}$ \\
\hline Vitreorana uranoscopa (Müller, 1924) & & $\mathrm{X}$ & $\mathrm{X}$ & $\mathrm{X}$ \\
\hline \multicolumn{5}{|l|}{ CERATOPHRYIDAE } \\
\hline Ceratophrys aurita (Raddi, 1823) & & $\mathrm{X}$ & $\mathrm{X}$ & $\mathrm{X}^{*}$ \\
\hline \multicolumn{5}{|l|}{ CRAUGASTORIDAE } \\
\hline Euparkerella tridactyla Izecksohn, 1988 & $\mathrm{X}$ & $\mathrm{X}$ & $\mathrm{X}$ & $\mathrm{X}$ \\
\hline Haddadus binotatus (Spix, 1824) & & $\mathrm{x}$ & $\mathrm{X}$ & $\mathrm{X}$ \\
\hline \multicolumn{5}{|l|}{ CYCLORAMPHIDAE } \\
\hline Cycloramphus fuliginosus Tschudi, 1838 & & $\mathrm{X}$ & $\mathrm{X}$ & $\mathrm{X}$ \\
\hline Thoropa aff. lutzi & & $\mathrm{X}$ & $\mathrm{X}^{*}$ & - \\
\hline Thoropa miliaris (Spix, 1824) & & $\mathrm{X}$ & $\mathrm{X}$ & $\mathrm{X}$ \\
\hline Thoropa petropolitana (Wandolleck, 1907) & & $\mathrm{X}$ & $\mathrm{X}$ & - \\
\hline Zachaenus carvalhoi Izecksohn, 1983 & $\mathrm{X}$ & $\mathrm{X}$ & $\mathrm{X}$ & $\mathrm{X}$ \\
\hline \multicolumn{5}{|l|}{ ELEUTHERODACTYLIDAE } \\
\hline $\begin{array}{l}\text { Adelophryne glandulata Lourenço-de-Moraes, Ferreira, Fouquet \& Bastos, } \\
2014\end{array}$ & $\mathrm{X}$ & $\mathrm{X}$ & $\mathrm{X}^{*}$ & - \\
\hline \multicolumn{5}{|l|}{ HEMIPHRACTIDAE } \\
\hline Fritziana aff. fissilis & & $\mathrm{X}$ & $\mathrm{X}^{*}$ & $\mathrm{X}^{*}$ \\
\hline Fritziana tonimi Walker, Gasparini, Haddad, 2016 & $\mathrm{X}$ & $\mathrm{X}$ & $\mathrm{X}^{*}$ & $\mathrm{X}^{*}$ \\
\hline Gastrotheca albolineata (Lutz \& Lutz, 1939) & & $\mathrm{X}$ & $\mathrm{X}$ & - \\
\hline Gastrotheca ernestoi Miranda-Ribeiro, 1920 & & $\mathrm{X}$ & - & - \\
\hline Gastrotheca megacephala Izecksohn, Carvalho-e-Silva \& Peixoto, 2009 & & $\mathrm{X}$ & - & - \\
\hline
\end{tabular}




\section{Species by Family}

\section{HYLIDAE}

Aparasphenodon brunoi Miranda-Ribeiro, 1920

Aplastodiscus cavicola (Cruz \& Peixoto, 1985)

Aplastodiscus aff. engenioi

Aplastodiscus weygoldti (Cruz \& Peixoto, 1987)

Boana albomarginata (Spix, 1824)

Boana albopunctata (Spix, 1824)

Boana crepitans (Wied-Neuwied, 1824)

Boana faber (Wied-Neuwied, 1821)

Boana pardalis (Spix, 1824)

Boana polytaenia (Cope, 1870)

Boana semilineata (Spix, 1824)

Bokermannohyla caramaschii (Napoli, 2005)

Dendropsophus berthalutzae (Bokermann, 1962)

Dendropsophus bipunctatus (Spix, 1824)

Dendropsophus branneri (Cochran, 1948)

Dendropsophus bromeliaceus Ferreira, Faivovich, Beard \& Pombal, 2015

Dendropsophus decipiens (Lutz, 1925)

Dendropsophus elegans (Wied-Neuwied, 1824)

Dendropsophus giesleri (Mertens, 1950)

Dendropsophus haddadi (Bastos \& Pombal, 1996)

Dendropsophus microps (Peters, 1872)

Dendropsophus minutus (Peters, 1872)

Dendropsophus ruschii (Weygoldt \& Peixoto, 1987)

Dendropsophus seniculus (Cope, 1868)

Itapotihyla langsdorffi (Duméril \& Bibron, 1841)

Ololygon arduous (Peixoto, 2002)

Ololygon argyreornata (Miranda-Ribeiro, 1926)

Ololygon cf. flavoguttata (Lutz \& Lutz, 1939)

Ololygon aff. heyeri

Ololygon heyeri Peixoto \& Weygoldt, 1986

Ololygon kautskyi Carvalho-e-Silva \& Peixoto, 1991

Phasmahyla exilis (Cruz, 1980)

Phrynomedusa marginata (Izecksohn \& Cruz, 1976)

Phyllodytes kautskyi Peixoto \& Cruz, 1988

Phyllodytes luteolus (Wied-Neuwied, 1824)

Phyllodytes aff. luteolus

Phyllomedusa burmeisteri Boulenger, 1882

Pithecopus aff. rohdei

Scinax alter (Lutz, 1973)

Scinax cuspidatus (Lutz, 1925)

Scinax eurydice (Bokermann, 1968)

Scinax fuscovarius (Lutz, 1925)

Scinax hayii (Barbour, 1909)

Scinax aff. perereca

Scinax cf. $x$-signatus (Spix, 1824)

\section{Type Our Almeida Rödder}

locality study et al. 2011 et al. 2007

\begin{tabular}{|c|c|c|c|}
\hline & X & - & X \\
\hline \multirow[t]{2}{*}{$X$} & $X$ & $X$ & X \\
\hline & $\mathrm{X}$ & - & - \\
\hline \multirow[t]{8}{*}{ X } & X & X & X \\
\hline & $X$ & $\mathrm{X}$ & X \\
\hline & $X$ & $\mathrm{X}$ & X \\
\hline & $\mathrm{X}$ & $\mathrm{X}$ & $\mathrm{X}$ \\
\hline & $\mathrm{X}$ & $\mathrm{X}$ & X \\
\hline & $\mathrm{X}$ & $\mathrm{X}$ & $X$ \\
\hline & $\mathrm{X}$ & $\mathrm{X}$ & - \\
\hline & X & $X$ & $X$ \\
\hline \multirow[t]{4}{*}{$X$} & $X$ & $X$ & X \\
\hline & $\mathrm{X}$ & X & $X$ \\
\hline & X & X & $X$ \\
\hline & X & X & X \\
\hline \multirow[t]{10}{*}{$\mathrm{X}$} & $\mathrm{X}$ & - & - \\
\hline & $X$ & $X$ & $\mathrm{X}$ \\
\hline & $\mathrm{X}$ & X & $X$ \\
\hline & $\mathrm{X}$ & X & $X$ \\
\hline & $X$ & X & $X$ \\
\hline & $\mathrm{X}$ & $\mathrm{X}$ & $X$ \\
\hline & $\mathrm{X}$ & X & $X$ \\
\hline & $\mathrm{X}$ & $\mathrm{X}$ & $X$ \\
\hline & $\mathrm{X}$ & X & $X$ \\
\hline & $X$ & X & $X$ \\
\hline \multirow[t]{4}{*}{$\mathrm{X}$} & $X$ & $X$ & $X$ \\
\hline & $\mathrm{X}$ & X & $X$ \\
\hline & $X$ & - & - \\
\hline & $X$ & - & - \\
\hline \multirow[t]{2}{*}{$X$} & $\mathrm{X}$ & $X$ & $X$ \\
\hline & $X$ & X & $X$ \\
\hline$X$ & $X$ & $\mathrm{X}$ & $X$ \\
\hline \multirow[t]{13}{*}{$X$} & $\mathrm{X}$ & X & X \\
\hline & $X$ & - & - \\
\hline & $\mathrm{X}$ & $X$ & $X$ \\
\hline & $\mathrm{X}$ & - & - \\
\hline & $X$ & $X$ & $\mathrm{X}$ \\
\hline & $\mathrm{X}$ & $\mathrm{X}^{*}$ & $\mathrm{X}^{*}$ \\
\hline & $X$ & $\mathrm{X}$ & $X$ \\
\hline & $\mathrm{X}$ & $\mathrm{X}$ & X \\
\hline & $X$ & $\mathrm{X}$ & $\mathrm{X}^{*}$ \\
\hline & $X$ & $\mathrm{X}$ & X \\
\hline & $X$ & X & $\mathrm{X}^{*}$ \\
\hline & $X$ & - & - \\
\hline & X & $X$ & $X$ \\
\hline
\end{tabular}




\begin{tabular}{|c|c|c|c|c|}
\hline Species by Family & Type & Our & Almeida & Rödder \\
\hline & locality & study & et al. 2011 & et al. 2007 \\
\hline Trachycephalus mesophaeus (Hensel, 1867) & & $\mathrm{X}$ & $\mathrm{X}$ & $\mathrm{X}$ \\
\hline Trachycephalus nigromaculatus Tschudi, 1838 & & $\mathrm{X}$ & $\mathrm{X}$ & $\mathrm{X}$ \\
\hline \multicolumn{5}{|l|}{ HYLODIDAE } \\
\hline Crossodactylus aff. gaudichaudii & & $\mathrm{X}$ & $\mathrm{X}$ & $\mathrm{X}^{*}$ \\
\hline Crossodactylus timbuhy Pimenta, Cruz \& Caramaschi, 2014 & $\mathrm{X}$ & $\mathrm{X}$ & $\mathrm{X}^{*}$ & $\mathrm{X}^{*}$ \\
\hline Hylodes cf. babax Heyer, 1982 & & $\mathrm{X}$ & $\mathrm{X}^{*}$ & $\mathrm{X}^{*}$ \\
\hline Hylodes lateristrigatus (Baumann, 1912) & & $\mathrm{X}$ & $\mathrm{X}$ & $\mathrm{X}$ \\
\hline Megaelosia apuana Pombal, Prado \& Canedo, 2003 & & $\mathrm{X}$ & $\mathrm{X}$ & $\mathrm{X}^{*}$ \\
\hline \multicolumn{5}{|l|}{ LEPTODACTYLIDAE } \\
\hline Crossodactylodes bokermanni Peixoto, 1983 & $\mathrm{X}$ & $\mathrm{X}$ & $\mathrm{X}$ & $\mathrm{X}$ \\
\hline Crossodactylodes izecksohni Peixoto, 1983 & $\mathrm{X}$ & $\mathrm{X}$ & $\mathrm{X}$ & $\mathrm{X}$ \\
\hline Leptodactylus cupreus Caramaschi, Feio \& São Pedro, 2008 & & $\mathrm{X}$ & $\mathrm{X}$ & - \\
\hline Leptodactylus fuscus (Schneider, 1799) & & $\mathrm{X}$ & $\mathrm{X}$ & $\mathrm{X}$ \\
\hline Leptodactylus aff. latrans (Steffen, 1815) & & $\mathrm{X}$ & $\mathrm{X}^{*}$ & $\mathrm{X}^{*}$ \\
\hline Leptodactylus aff. spixi & & $\mathrm{X}$ & $\mathrm{X}^{*}$ & $\mathrm{X}^{*}$ \\
\hline Physalaemus crombiei Heyer \& Wolf, 1989 & $\mathrm{X}$ & $\mathrm{x}$ & $\mathrm{X}$ & $\mathrm{X}$ \\
\hline Physalaemus cuvieri Fitzinger, 1826 & & $\mathrm{x}$ & $\mathrm{X}$ & $\mathrm{X}$ \\
\hline Physalaemus maculiventris (Lutz, 1925) & & $\mathrm{x}$ & $\mathrm{X}$ & - \\
\hline Physalaemus cf. olfersii (Lichtenstein \& Martens, 1856) & & $\mathrm{X}$ & $\mathrm{X}^{*}$ & $\mathrm{X}^{*}$ \\
\hline \multicolumn{5}{|l|}{ MICROHYLIDAE } \\
\hline Chiasmocleis capixaba Cruz, Caramaschi \& Izecksohn, 1997 & & $\mathrm{X}$ & - & - \\
\hline Chiasmocleis schubarti Bokermann, 1952 & & $\mathrm{X}$ & - & $\mathrm{X}$ \\
\hline Myersiella microps (Duméril \& Bibron, 1841) & & $\mathrm{X}$ & $\mathrm{X}$ & $\mathrm{X}$ \\
\hline \multicolumn{5}{|l|}{ ODONTOPHRYNIDAE } \\
\hline Macrogenioglottus alipioi Carvalho, 1946 & & $\mathrm{X}$ & $\mathrm{X}$ & $\mathrm{X}$ \\
\hline Proceratophrys boiei (Wied-Neuwied, 1824) & & $\mathrm{X}$ & $\mathrm{X}$ & $\mathrm{X}$ \\
\hline Proceratophrys laticeps Izecksohn \& Peixoto, 1981 & & $\mathrm{X}$ & $\mathrm{X}$ & $\mathrm{X}$ \\
\hline Proceratophrys moehringi Weygoldt \& Peixoto, 1985 & $\mathrm{X}$ & $\mathrm{X}$ & $\mathrm{X}$ & $\mathrm{X}$ \\
\hline Proceratophrys paviotii Cruz, Prado \& Izecksohn, 2005 & $\mathrm{X}$ & $\mathrm{X}$ & $\mathrm{X}$ & $\mathrm{X}$ \\
\hline Proceratophrys phyllostomus Izecksohn, Cruz \& Peixoto, 1999 & & $\mathrm{X}$ & $\mathrm{X}$ & $\mathrm{X}$ \\
\hline Proceratophrys schirchi (Miranda-Ribeiro, 1937) & & $\mathrm{X}$ & $\mathrm{X}$ & $\mathrm{X}$ \\
\hline \multicolumn{5}{|l|}{ PIPIDAE } \\
\hline Pipa aff. carvalhoi & & $\mathrm{X}$ & $\mathrm{X}^{*}$ & $\mathrm{X}^{*}$ \\
\hline \multicolumn{5}{|l|}{ RANIDAE } \\
\hline Lithobates catesbeianus (Shaw, 1802) & & $\mathrm{X}$ & - & - \\
\hline \multicolumn{5}{|l|}{ SIPHONOPIDAE } \\
\hline Siphonops annulatus (Mikan, 1822) & & $\mathrm{X}$ & - & - \\
\hline Siphonops hardyi Boulenger, 1888 & & $\mathrm{X}$ & - & - \\
\hline
\end{tabular}

with 47 species (43\%), Brachycephalidae with 11 species (10\%), and Leptodactylidae with 10 species (9\%). Santa Teresa is currently the type locality for 23 species (20\%) (Table 1). So far, four species (3\%) are only found in Santa Teresa such as Crossodactylodes izecksohni, Crossodactylus timbuhy, Ischnocnema colibri and Ischnocnema epipeda. The species density of Santa Teresa is 0.16 species per $\mathrm{km}^{2}$ (i.e., 108 species $/ 683 \mathrm{~km}^{2}$ ). 


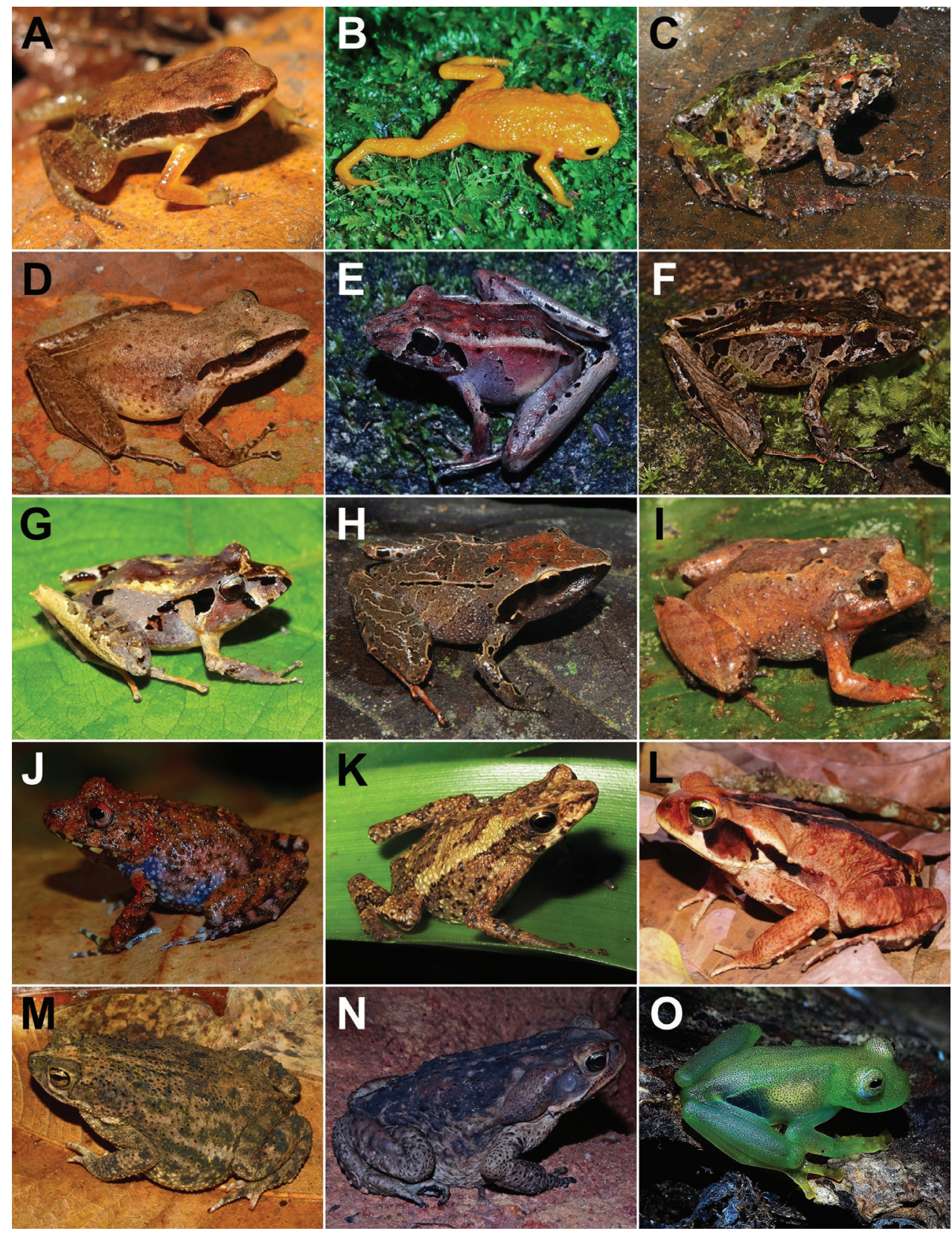

Figure 2. Amphibians from Santa Teresa: A Allobates capixaba B Brachycephalus alipioi C Ischnocnema abdita $\mathbf{D}$ Ischnocnema colibri $\mathbf{E}$ Ischnocnema cf. nasuta $\mathbf{F}$ Ischnocnema aff. guentheri $\mathbf{G}$ Ischnocnema oea $\mathbf{H}$ Ischnocnema gr. parva sp. new 1 I Ischnocnema gr. parva sp. new $2 \mathbf{J}$ Ischnocnema verrucosa $\mathbf{K}$ Dendrophryniscus carvalhoi $\mathbf{L}$ Rhinella crucifer $\mathbf{M}$ Rhinella granulosa $\mathbf{N}$ Rhinella diptycha $\mathbf{O}$ Vitreorana aff. eurygnatha. Photographs by JFR Tonini (A), CN Fraga (B), RB Ferreira (C, D, H, I, K), AT Mônico (E, G, J, K, L, $\mathbf{M}, \mathbf{N}, \mathbf{O})$, T Silva-Soares (F). 


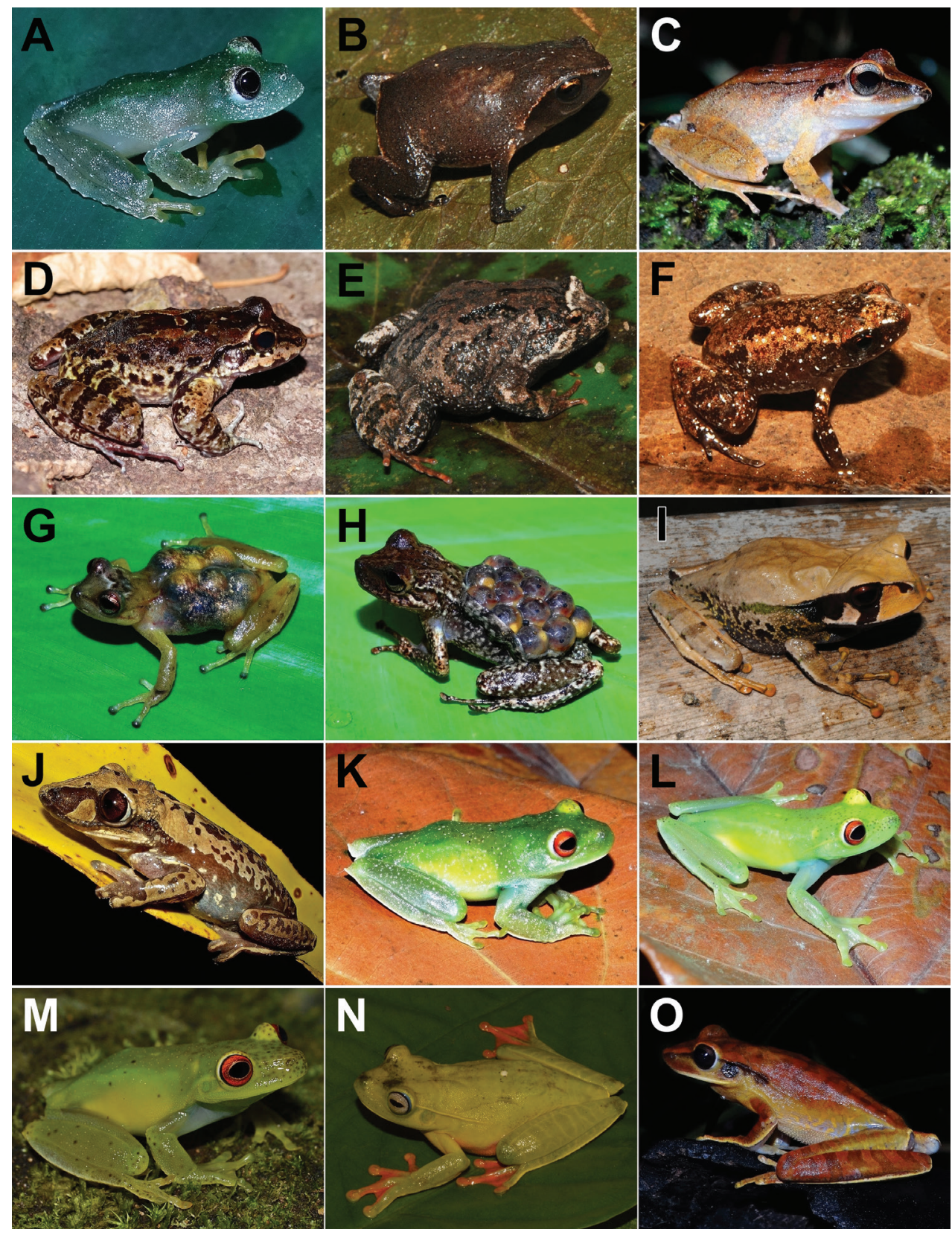

Figure 3. Amphibians from Santa Teresa: A Vitreorana uranoscopa B Euparkerella tridactyla C Haddadus binotatus $\mathbf{D}$ Thoropa miliaris $\mathbf{E}$ Zachaenus carvalhoi $\mathbf{F}$ Adelophryne glandulata $\mathbf{G}$ Fritziana aff. fissilis $\mathbf{H}$ Fritziana tonimi I Gastrotheca megacephala $\mathbf{J}$ Aparasphenodon bruno $\mathbf{K}$ Aplastodiscus cavicola $\mathbf{L}$ Aplastodiscus aff. engenioi $\mathbf{M}$ Aplastodiscus weygoldti $\mathbf{N}$ Boana albomarginata $\mathbf{O}$ Boana albopunctata. Photographs by AT Mônico (A, C, D, G, H, K, L, O), RB Ferreira (B, E, F, I, N), C Zocca (J), T Silva-Soares (M). 


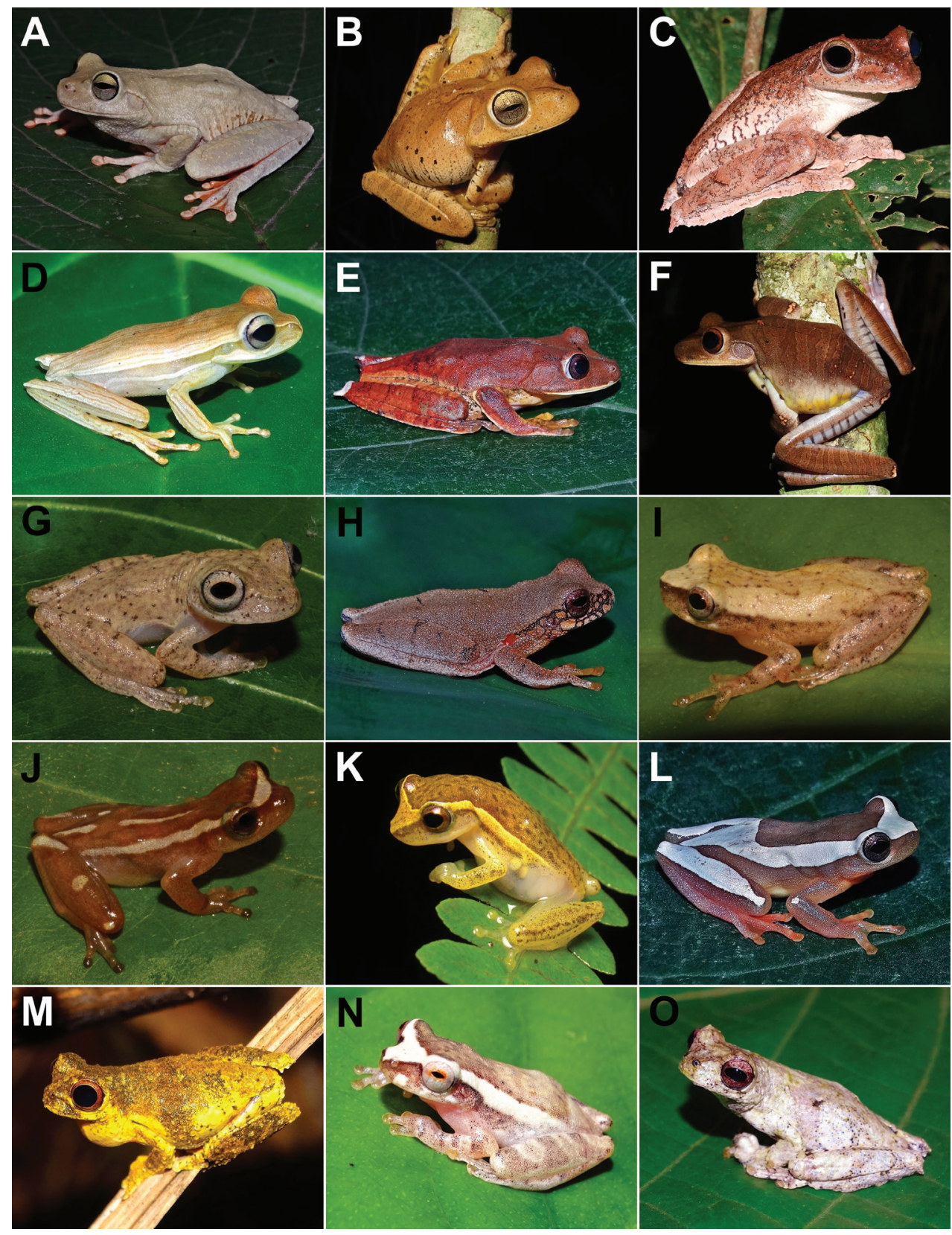

Figure 4. Amphibians from Santa Teresa: A Boana crepitans B Boana faber C Boana pardalis D Boana polytaenia E Boana semilineata $\mathbf{F}$ Bokermannohyla caramaschii $\mathbf{G}$ Dendropsophus berthalutzae $\mathbf{H}$ Dendropsophus bipunctatus I Dendropsophus branneri J Dendropsophus bromeliaceus $\mathbf{K}$ Dendropsophus decipiens $\mathbf{L}$ Dendropsophus elegans $\mathbf{M}$ Dendropsophus giesleri $\mathbf{N}$ Dendropsophus haddadi $\mathbf{O}$ Dendropsophus microps. Photographs by AT Mônico (A, C, D, E, F, H, L, M, N, O), RB Ferreira (B, G, I, J), ET Silva (K). 


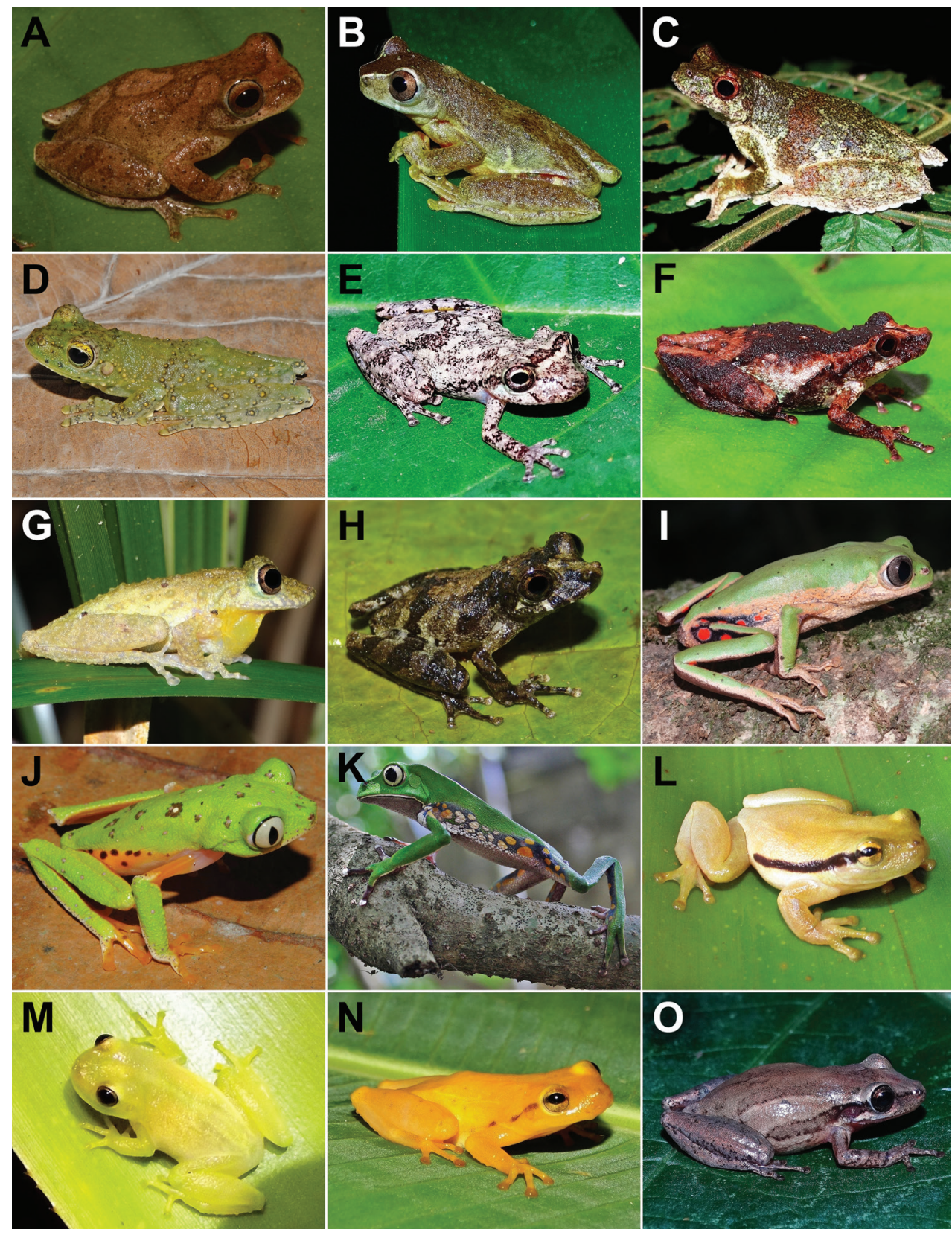

Figure 5. Amphibians from Santa Teresa: A Dendropsophus minutus B Dendropsophus ruschii C Dendropsophus seniculus $\mathbf{D}$ Itapotihyla langsdorffi $\mathbf{E}$ Ololygon arduous $\mathbf{F}$ Ololygon argyreornata $\mathbf{G}$ Ololygon heyeri H Ololygon kautskyi I Pithecopus aff. rohdei J Phasmahyla exilis $\mathbf{K}$ Phyllomedusa burmeisteri $\mathbf{L}$ Phyllodytes kautskyi $\mathbf{M}$ Phyllodytes luteolus $\mathbf{N}$ Phyllodytes aff. luteolus $\mathbf{O}$ Scinax alter. Photographs by RB Ferreira (A, D, J), AT Mônico (B, C, E, F, G, I, K, N, O), T Silva-Soares (H), CZ Zocca (L, M). 


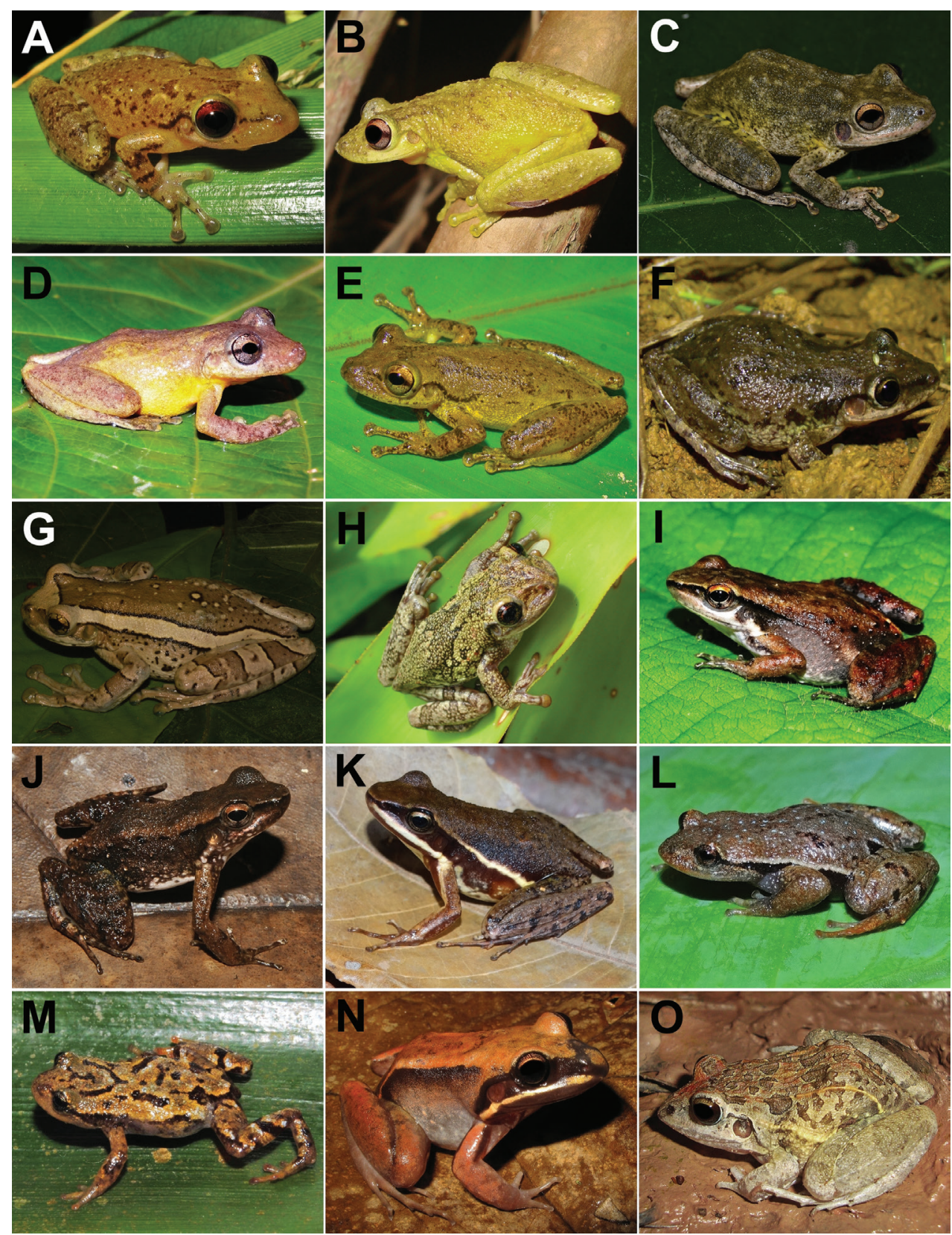

Figure 6. Amphibians from Santa Teresa: A Scinax cuspidatus B Scinax eurydice C Scinax fuscovarius D Scinax hayii E Scinax aff. perereca $\mathbf{F}$ Scinax cf. $x$-signatus $\mathbf{G}$ Trachycephalus mesophaeus $\mathbf{H}$ Trachycephalus nigromaculatus $\mathbf{I}$ Crossodactylus aff. gaudichaudii $\mathbf{J}$ Crossodactylus timbuhy $\mathbf{K}$ Hylodes lateristrigatus $\mathbf{L}$ Crossodactylodes bokermanni $\mathbf{M}$ Crossodactylodes izecksohni $\mathbf{N}$ Leptodactylus cupreus $\mathbf{O}$ Leptodactylus fuscus. Photographs by ET Silva (A, E, F), CZ Zocca $(\mathbf{B}, \mathbf{O})$, T Silva-Soares $(\mathbf{C})$, AT Mônico (D, I, K, L), RB Ferreira $(\mathbf{G}, \mathbf{H}, \mathbf{J}, \mathbf{M})$, JFR Tonini $(\mathbf{N})$. 


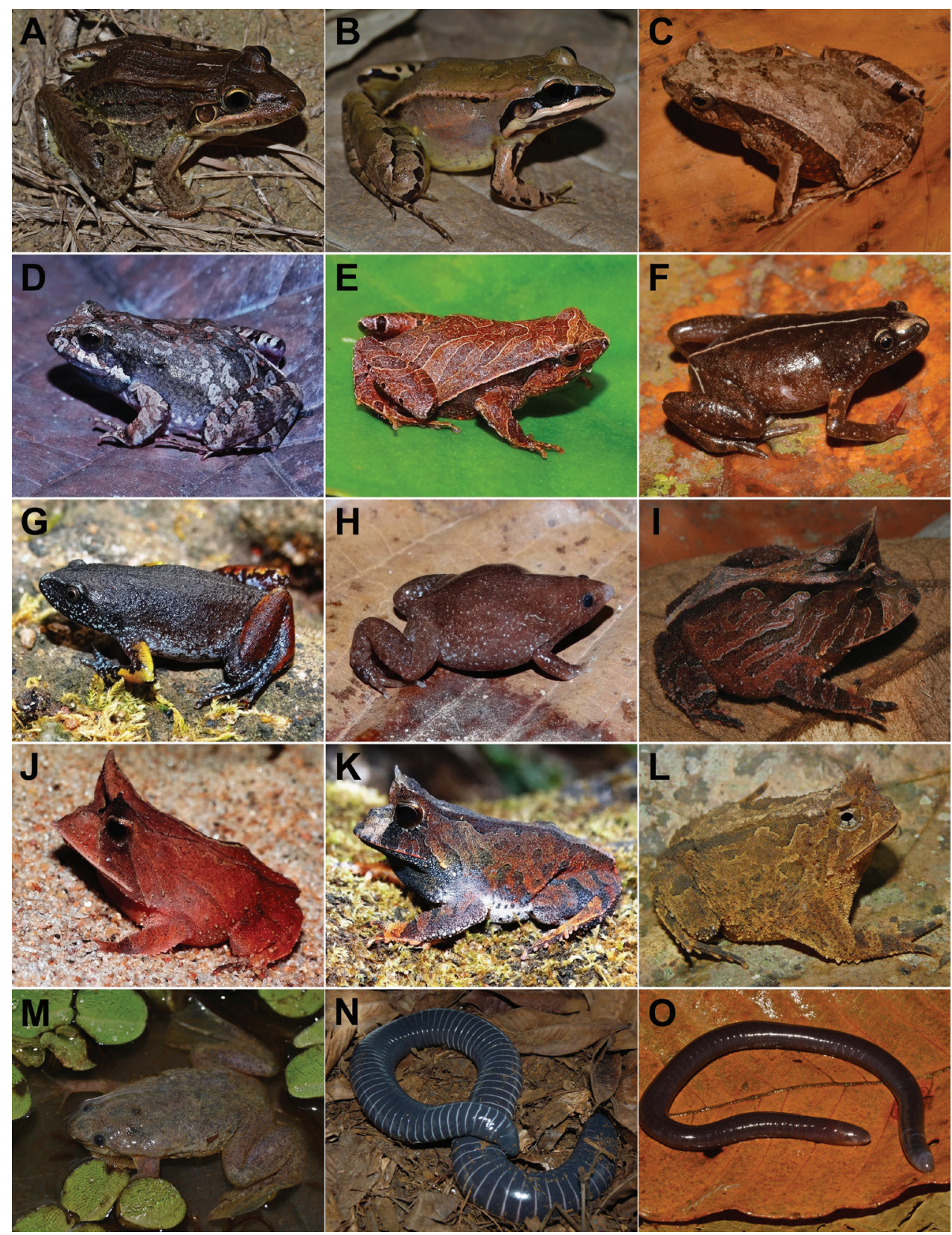

Figure 7. Amphibians from Santa Teresa: A Leptodactylus aff. Latrans B Leptodactylus aff. spixi C Physalaemus crombiei D Physalaemus cuvieri $\mathbf{E}$ Physalaemus maculiventris $\mathbf{F}$ Chiasmocleis capixaba $\mathbf{G}$ Chiasmocleis schubarti $\mathbf{H}$ Myersiella microps $\mathbf{I}$ Proceratophrys boiei $\mathbf{J}$ Proceratophrys laticeps $\mathbf{K}$ Proceratophrys paviotii $\mathbf{L}$ Proceratophrys schirchi $\mathbf{M}$ Pipa aff. carvalhoi $\mathbf{N}$ Siphonops annulatus $\mathbf{O}$ Siphonops hardyi. Photographs by T Silva-Soares (A, B, L, M, N), AT Mônico (C, D, E, G, H, J, K), RB Ferreira (F, I, O). 
Compared to previous anuran lists for Santa Teresa, we added 14 species, removed 17 previously reported species, and re-determined 14 species based on recent taxonomic rearrangements. Out of the 14 added species, 11 (79\%) were first recorded during our fieldwork and specimen examination, two (14\%) records were from the literature, and one (7\%) new record was from pers. comm. (Gastrotheca ernestoi; MT Rodrigues, field number MTR 34695).

Fourteen species classified to morphotypes are new species, such as Aplastodiscus aff. eugenioi (M Mongin, pers. comm.), Brachycephalus aff. didactylus (TSS, in. prep.), Crossodactylus aff. gaudichaudii (R Montesinos, in. prep.), Fritziana aff. fissilis (RBF, pers. obs.), Ischnocnema aff. parva sp. 1 (CAG Cruz, in. prep.), Ischnocnema aff. parva sp. 2 (TSS, in. prep.), Leptodactylus aff. spixi (L Nascimento, in. prep.), Ololygon aff. heyeri (J Lacerda, pers. comm.), Phyllodytes aff. luteolus (ATM, in. prep.), Pipa aff. carvalhoi (PV Scherrer, in. prep.), Pithecopus aff. rohdei (D Baêta, pers. comm.), Scinax aff. perereca (TSS, pers. comm.), Thoropa aff. lutzi (CL Assis, pers. comm.), and Vitreorana aff. eurygnatha (R Pontes, in. prep.).

\section{Discussion}

The current number of 106 anuran species for Santa Teresa is remarkable, and represents 78\% of the 136 species listed for Espírito Santo state (Almeida et al. 2011, RossaFeres et al. 2017), 10\% of the 1,080 species listed for Brazil (Segalla et al. 2016), and $1.5 \%$ of the 7,068 species listed worldwide (AmphibiaWeb 2019). To date, the species density (i.e., 0.16 species per $\mathrm{km}^{2}$ ) is one of the highest in the world at regional scale. For instance, Yasuní National Park in Ecuador has 0.015 species per $\mathrm{km}^{2}$ (i.e., 150 species $/ 9,820 \mathrm{~km}^{2}$; Bass et al. 2010); Tambopata in southern Peru has 0.06 species per $\mathrm{km}^{2}$ (i.e., 99 species $/ 1,600 \mathrm{~km}^{2}$; Doan and Arriaga 2002); Iquitos region of northern Loreto in Peru has 0.012 species per $\mathrm{km}^{2}$ (i.e., 141 species $/ 11,310 \mathrm{~km}^{2}$; IUCN 2008, Rodríguez and Duellman 1994); and Leticia in Colombia has 0.13 species per $\mathrm{km}^{2}$ (i.e., 123 species $/ 927 \mathrm{~km}^{2}$; Lynch 2005). Several other localities across the Atlantic Forest also have remarkable amphibian richness at local scales. For example, Reserva Biológica de Paranapiacaba in Sao Paulo state has 20.5 species per $\mathrm{km}^{2}$ (69 species/3.36 $\mathrm{km}^{2}$; Verdade et al. 2009); Fazenda Vista Bela in Bahia state has 7.3 species per $\mathrm{km}^{2}$ (34 species $/ 4.65 \mathrm{~km}^{2}$; Silvano and Pimenta 2003); and Reserva Particular do Patrimônio Natural Serra Bonita has 4 species per $\mathrm{km}^{2}$ (80 species $/ 20 \mathrm{~km}^{2}$; Dias et al. 2014). We acknowledge that amphibian richness per area represents just a first approximation for practical spatial comparisons and that the lack of adequate surveys in more unexplored diverse regions (e.g., Indonesia, New Guinea, and the Congo Basin) may reveal remarkable amphibian richness. So far, Brazil's Atlantic Forest and the northwest Amazon are considered the world's greatest amphibian diversity on a landscape scale (Young et al. 2004, Bass et al. 2010).

The two species of Gymnophiona (Siphonops annulatus and S. hardyi) were found during our fieldwork but have been reported previously for Santa Teresa (Caramaschi 
et al. 2004, Maciel et al. 2009). The former has a wide distribution in South America from Colombia to Argentina (Frost 2018). The latter has a more restricted distribution in southeastern of Brazil (Maciel et al. 2009, Frost 2018). Caecilians are difficult to sample due to the subterranean or aquatic habits (Oommen et al. 2000, Maciel and Hoogmoed 2011). Although amphibians are dramatically declining (Stuart et al. 2004), the conservation status of caecilians is largely unknown due to the lack of information on their biology, ecology and natural history (Wilkinson and Nussbaum 1999, Oommen et al. 2000, Gower and Wilkinson 2005). It is likely more species of caecilians will be recorded in Santa Teresa if the use of sampling methods specific for these taxa is applied in the field.

Our fieldwork since 2005 in Santa Teresa has made notable contributions toward the knowledge of local amphibians. It has resulted in the description of three new species for the municipality (i.e., Adelophryne glandulata in Lourenço-de-Moraes, Ferreira, Fouquet, Bastos 2014, Dendropsophus bromeliaceus in Ferreira, Faivovich, Beard, Pombal 2015, and Ischnocnema colibri in Taucce, Canedo, Parreiras, Drummond, Nogueira-Costa, Haddad 2018). Furthermore, our fieldwork found individuals of 13 morphospecies that are currently under formal description (i.e., Aplastodiscus aff. eugenioi, Brachycephalus aff. didactylus, Crossodactylus aff. gaudichaudii, Fritziana aff. fissilis, Ischnocnema aff. parva sp. 1, Ischnocnema aff. parva sp. 2, Leptodactylus aff. spixi, Ololygon aff. heyeri, Phyllodytes aff. luteolus, Pipa aff. carvalhoi, Pithecopus aff. rohdei, Scinax aff. perereca, and Vitreorana aff. eurygnatha). The discovery of new species, morphospecies, and new records for Santa Teresa may be due to our sampling in remote forested areas and rocky outcrops through both visual bromeliad surveys and active leaf-litter searches (Ferreira et al. 2016).

Our species list resolved some differences between the previous species lists of Santa Teresa, which had disagreements on 11 species (e.g., Rödder et al. 2007, Almeida et al. 2011). We confirmed that Chiasmocleis schubarti occurs in Santa Teresa based on several individuals sampled in the Reserva Biológica Augusto Ruschi, whereas Almeida et al. (2011) challenged previous records of this species listed in Cruz et al. (1997) and Rödder et al. (2007). We also confirmed the presence of Aparasphenodon brunoi and Trachycephalus nigromaculatus reported in Santa Teresa at the buffer zone of the Parque Municipal do Goiapaba-Açu (Ramos and Gasparini 2004). Almeida et al. (2011) challenged the record of Rhinella hoogmoedi referring to the species as Rhinella gr. margaritifer, because the former species was not mentioned in Rödder et al. (2007). We agree with Almeida et al. (2011) regarding the exclusion of several species from Rödder et al. (2007), such as Bokermannohyla aff. nanuzae (MBML 4528 corresponds to B. caramaschii), Dendrophryniscus sp. (MBML 3841 corresponds to D. carvalhoi), Ischnocnema cf. juipoca (MBML 5737 corresponds to I. abdita), I. lactea (MBML 1143 corresponds to I. abdita), Physalaemus aguirrei (MBML 2803-04 correspond to P. cf. olfersii), and Proceratophrys appendiculata (MBML 1154 corresponds to P. schirchii). Rödder et al. (2007) and Almeida et al. (2011) listed Leptodactylus natalensis for Santa Teresa but the voucher specimens (MBML 3909-10) were misidentified and actually refer to individuals of $L$. aff. spixi. Rödder et al. (2007) listed Allobates cf. olfersioides 
following Verdade and Rodrigues (2007) who placed $A$. capixaba as synonym of $A$. olfersioides. Studies on Allobates indicate A. capixaba is a valid taxon (e.g., Bokermann 1967; Forti et al. 2017), which agrees with Almeida et al. (2011). Fieldwork should be conducted in the vicinities of Santa Teresa to confirm the presence of Brachycephalus alipioi. This species has not been found in Santa Teresa since 1952 when the municipality was larger than it is today (Pombal and Gasparini 2006).

The wide elevational range of Santa Teresa $(-120-1099 \mathrm{~m}$ a.s.l.) partially explains the high richness of amphibian species. Species typical of both Atlantic Forest lowlands (e.g., Allobates capixaba, Chiasmocleis schubarti, C. capixaba, Dendropsophus bipunctatus, Ololygon argyreornata) and highlands (e.g., Aplastodiscus cavicola, Bokermannohyla caramaschii, Dendropsophus ruschii) occur in Santa Teresa, which suggest that the elevational gradient influences species composition. The high amphibian diversity also may be related to edaphic and topographic heterogeneity, which is known to cause speciation in many Atlantic Forest species occurring in mountainous areas (Carnaval et al. 2014). The high altitude and proximity to the Atlantic Ocean favors frequent orographic rain, which contribute to the meeting the reproductive requirements of amphibians. It is worth highlighting that Santa Teresa is one of the most sampled regions for amphibians in the Atlantic Forest (Rödder et al. 2007, Almeida et al. 2011, Zocca et al. 2014, Ferreira et al. 2016). About 3,800 anuran specimens collected in Santa Teresa were found housed in Brazilian collections (ET Silva, pers. obs.). This high sampling effort, which is comparable to only a few localities in the Atlantic Forest, may also account for such high species richness.

\section{Conservation remarks}

Amphibians from Santa Teresa have faced several anthropogenic disturbances over the last couple of decades. The first report on amphibian declines for Santa Teresa was in 1989 (see Weygoldt 1989). During long-term sporadic samplings (i.e., 1975 and 1988), Weygoldt (1989) reported the decline and possible disappearances of eight species (updated taxonomy: Allobates capixaba, Crossodactylus aff. gaudichaudii, C. timbuhy, Cycloramphus fuliginosus, Hylodes lateristrigatus, H. cf. babax, Phasmahyla exilis, and Vitreorana aff. eurygnatha). To our knowledge, Cycloramphus fuliginosus and $\mathrm{Hy}$ lodes cf. babax have not been recorded after Weygoldt (1989). Additionally, Thoropa petropolitana, a frog not mentioned by Weygoldt (1989) has disappeared with no recent records along its entire range (Haddad et al. 2016). Several potential causes of these declines were mentioned by Weygoldt (1989), such as pollution (acid rain and pesticides), long-term climatic changes, and epidemic diseases. Weygoldt (1986) mentioned that Crossodactylus cf. dispar (currently C. timbuhy) was rare in Santa Teresa and later reported its decline. However, during our surveys we easily found this species on creeks across Santa Teresa. We cannot assess whether species declines are actually happening in Santa Teresa because only long-term and species-specific studies can precisely understand population trends. 
Over the decades, we have noted population disappearances of anurans in Santa Teresa. The construction of condominiums and vacation ranches has intensified over the last decade and consequently increased deforestation of primary forest. We have also observed the expansion of the non-native Eucalyptus spp. plantations near primary and secondary forests and the replacement of coffee plantations. Another unmeasured concern is the increasing record of morphological anuran deformities, which is likely a result of pesticides used on crops (e.g., Mônico et al. 2016), including inside the buffer zone of the largest forest reserve (i.e., Reserva Biológica Augusto Ruschi; pers. obs.). The report of the invasive frog, Lithobates catesbeianus, in Santa Teresa (see Ferreira and Lima 2012) should be further evaluated to monitor its establishment, and possible spread and impacts. We emphasize the need to sample the surroundings of the nearby breeding farms of L. catesbeianus. Studies have shown that non-native L. catesbeianus can be voracious predators of native anurans and vectors of diseases (Schloegel et al. 2010, Silva et al. 2011, Boelter et al. 2012).

The landscape configuration of Santa Teresa does not safeguard the maintenance of amphibian reproduction outside protected reserves because forests on private properties are mostly restricted to hilltops and non-natural matrix habitats occupy most valleys. Because water-body breeding species migrate toward reproductive habitats in the valleys, these species face severe threats, such as the risk of predation and desiccation (Becker et al. 2007, Ferreira et al. 2016). In addition, pollution of creeks and streams further strengthen conservation concern of lotic body breeders. We reinforce the need of studies focused on the threats amphibians are facing in the region to provide knowledge for conservationists and reserves managers to safeguard the local diversity.

Santa Teresa is an important hotspot for amphibian conservation due to its high richness and number of endemic species. The discovery of several new species further emphasizes the importance of this mountainous region for amphibian conservation. Even though Santa Teresa and its surrounding areas in southeastern Brazil are one of the most sampled regions in the Atlantic Forest, the region still harbors numerous remote areas that have not yet been sampled for frogs (e.g., Almeida et al. 2011). Forests on private properties are also important for preserving amphibian diversity in the area (Ferreira et al. 2016). In addition, private properties may function as forest corridors for dispersing and migrating species. We suggest that a program to stimulate the creation of private-owner reserves and ecotourism activities should be implemented in this region. Finally, we have been developing outreach activities (e.g., Bromeligenous Project) with the local farmers, aiming to minimize the anthropogenic effects on anurans. Nevertheless, there is a strong need for a long-term outreach program in the local schools and in the farmlands to protect these forest areas in the future.

\section{Acknowledgements}

We dedicated this manuscript to Rogério L Teixeira who was born and raised in Santa Teresa and dedicated decades sampling frogs and mentoring herpetologists. We thank 
Bromeligenous Project for field support; landowners for allowing access to their properties; Instituto Nacional da Mata Atlântica for logistic support. We are grateful to Cecilia Waichert, Francys Lacchine, Gustavo Milanezi, Jandyra Zocca Zandomenico, Juliano Saich, Lamara P Barbosa, Namany Lourpen, Paulo R Jesus, Randerson LB Ferreira for field sampling. We especially thank Carlos A Cruz, Clarissa Canedo, Clodoaldo L Assis, Délio Baeta, Gustavo Prado, João V Lacerda, Juliana Kirchmeyer, Juliana Peres, Leo Malagoli, Marcele Mongin, Marco A Peixoto, Miguel Trefault Rodrigues, Paulo V Scherrer, Pedro Taucce, Rafael Pontes, Raquel Montesinos, and Victor Dill for discussions on species identification. Sampling permits were issued by Instituto Chico Mendes de Conservação da Biodiversidade (ICMBio, permits 28607, 50402, and 63575) and Institutional Animal Care and Use Committee (IACUC-USU, permit 2002). RBF (0823/FCLF (001/1774502), and CZZ (001/1700071) thank Coordenação de Aperfeiçoamento Pessoal de Nível Superior - Brasil (CAPES) for scholarships. RBF, ATM, ETS and TSS thank Conselho Nacional de Desenvolvimento Científico e Tecnológico (CNPq: 430195/2018, 304374/2016-4, 141569/2014-0, and 454789/2015-7) for scholarships. JFRT thanks CAPES/Science without Borders and David Rockefeller Center for Latin Studies/Harvard University for scholarships. This research was supported by the Utah Agricultural Experiment Station, Utah State University, and approved as journal paper number 9217 and by Harvard Open Access Equity Fund (HOPE).

\section{References}

Almeida AP, Gasparini JL, Peloso PLV (2011) Frogs of the state of Espírito Santo, southeastern Brazil - The need for looking at the coldspots. CheckList 7(4): 542-560. https://doi. org/10.15560/7.4.542

Alvares CA, Stape JL, Sentelhas PC, Gonçalves JLM, Sparoveck G (2013) Koppen's climate classification map for Brazil. Meteorologische Zeitschrift 22: 711-728. https://doi. org/10.1127/0941-2948/2013/0507

American and Veterinary Medical Association (2013) Guidelines for the euthanasia of animals. AVMA, Schaumberg, IL.

AmphibiaWeb (2019) University of California, Berkeley, CA. http://amphibiaweb.org [Accessed on 20 May 2019]

Bass MS, Finer M, Jenkins CN, Kreft H, Cisneros-Heredia DF, McCracken SF, Pitman NCA, English PH, Swing K, Villa G, Fiore AD, Voigt CC, Kunz TH (2010) Global Conservation Significance of Ecuador's Yasuní National Park. PloS ONE 5: e8767. https://doi. org/10.1371/journal.pone.0008767

Becker CG, Fonseca CR, Haddad CFB, Batista RF, Prado PI (2007) Habitat split and the global decline of amphibians. Science 318: 1775-1777. https://doi.org/10.1126/science. 1149374

Boelter RA, Kaefer IL, Both C, Cechin S (2012) Invasive bullfrogs as predators in a Neotropical assemblage: What frog species do they eat? Animal Biology 62: 397-408. https://doi. org/10.1163/157075612X634111 
Bokermann WCA (1967) Novas espécies de Phyllobates do leste e sudeste brasileiro (Anura, Dendrobatidae). Revista Brasileira de Biologia 27: 349-353.

Brasil (1983) Departamento Nacional de Produção Mineral. Projeto RADAM. V32. Folhas SF23/24 Rio de Janeiro/Vitória, Rio de Janeiro.

Brown KS Jr, Freitas AVL (2000) Atlantic Forest butterflies: indicators for landscape conservation. Biotropica 32: 934-956. https://doi.org/10.1111/j.1744-7429.2000.tb00631.x

Campos FS, Lourenço-de-Moraes R, Llorente GA, Solé M (2017) Cost-effective conservation of amphibian ecology and evolution. Science Advances 3(6): e1602929. https://doi. org/10.1126/sciadv.1602929

Campos FS, Lourenço-de-Moraes R (2017) Amphibians from the mountains of the Serra do Mar Coastal Forest, Brazil. Herpetology Notes 10: 547-560.

Caramaschi U, Rodrigues MT, Carvalho-e-Silva SP, Cruz CAG (2004) Siphonops hardyi. IUCN Red List of Threatened Species. http://www.iucnredlist.org [Acessed on 17 April 2009]

Carnaval AC, Waltari E, Rodrigues MT, Rosauer D, VanDerWal J, Damasceno R, Prates I, Strangas M, Spanos Z, Rivera D, Pie MR, Firkowski CR, Bornschein MR, Ribeiro LF, Moritz C (2014) Prediction of phylogeographic endemism in an environmentally complex biome. Proceedings of the Royal Society B. Biological Science 281: 20141461. https://doi. org/10.1098/rspb.2014.1461

Carvalho T, Becker CG, Toledo LF (2017) Historical amphibian declines and extinctions in Brazil linked to chytridiomycosis. Proceedings of the Royal Society Biological Sciences 284: 20162254. https://doi.org/10.1098/rspb.2016.2254

CEBEA/CFMV CdÉBeB-EA (2013) Guia brasileiro de boas práticas para eutanásia de animais. Conselho Federal de Medicina Veterinária do Brasil, Brasília, 62 pp.

Cruz CAG, Caramaschi U, Izecksohn E (1997) The genus Chiasmocleis Méhely 1904 (Anura, Microhylidae) in the Atlantic Rain Forest of Brazil, with description of three new species. Alytes 15: 49-71.

Dias I, Medeiros T, Vila Nova M, Solé M (2014) Amphibians of Serra Bonita, southern Bahia: a new hotpoint within Brazil's Atlantic Forest hotspot. ZooKeys 449: 105-130. https://doi. org/10.3897/zookeys.449.7494

Doan TM, Arriaga WA (2002) Microgeographic variation in species composition of the herpetofaunal communities of Tambopata Region, Peru. Biotropica 34: 101-117. https://doi. org/10.1111/j.1744-7429.2002.tb00246.x

Dodd Jr CK (2010) Diversity and similarity. In: Dodd Jr CK (Ed.) Amphibian Ecology and Conservation. A Handbook of Techniques, Oxford University Press, Oxford, 321-337.

Ferreira RB, Faivovich J, Beard KH, Pombal JP (2015) The first bromeligenous species of Dendropsophus (Anura, Hylidae) from Brazil's Atlantic Forest. PloS ONE 10: 1-21. https://doi. org/10.1371/journal.pone.0142893

Ferreira RB, Lima C (2012) Anuran hotspot at Brazilian Atlantic rainforest invaded by the non-native Lithobates catesbeianus Shaw 1802 (Anura, Ranidae). North-Western Journal of Zoology 8: 386-389.

Ferreira RB, Beard KH, Crump ML (2016) Breeding guild determines frog distributions in response to edge effects and habitat conversion in the Brazil's Atlantic Forest. PloS ONE 11: e0156781. https://doi.org/10.1371/journal.pone.0156781 
Forti LR, da Silva TRA, Toledo LF (2017) The acoustic repertoire of the Atlantic Forest Rocket Frog and its consequences for taxonomy and conservation (Allobates, Aromobatidae). ZooKeys 692: 141-153. https://doi.org/10.3897/zookeys.692.12187

Frost DR (2018) Amphibian Species of the World: an Online Reference. Version 6.0. American Museum of Natural History, New York. http://research.amnh.org/herpetology/amphibia/ index.html [accessed on 15 August 2017]

Gower DJ, Wilkinson M (2005) The conservation biology of caecilians. Conservation Biology 19: 45-55. https://doi.org/10.1111/j.1523-1739.2005.00589.x

Haddad CFB, Segalla MV, Bataus YSL, Caramaschi U (2016) Avaliação do Risco de Extinção de Thoropa petropolitana (Wandolleck 1907). http://www.icmbio.gov.br/portal/faunabrasileira/ estado-de-conservacao/7521-anfibios-thoropa-petropolitana [Accessed on 17 April 2017]

Heyer WR, Rand AS, Cruz CAG, Peixoto OL (1988) Decimations, extinctions, and colonizations of frog populations in Southeast Brazil and their evolutionary implications. Biotropica 20: 230-235. https://doi.org/10.2307/2388238

IUCN (2008) Guidelines for using the IUCN red list categories and criteria. Version 11. Prepared by the Standards and Petitions Subcommittee. www.iucnredlist.org [accessed on 15 August 2017]

Lourenço-de-Moraes R, Ferreira RB, Fouquet A, Bastos R (2014) A new diminutive frog of the genus Adelophryne Hoogmoed and Lescure 1984 (Amphibia, Anura, Eleutherodactylidae) from the Atlantic forest of Espírito Santo, Brazil. Zootaxa 3846: 348-360. https://doi. org/10.11646/zootaxa.3846.3.2

Lourenço-de-Moraes R, Campos FS, Ferreira RB, Beard K, Solé M, Bastos RP (2019) Back to the future: the role of climatic refuges in amphibians hotspot Atlantic Forest. Biodiversity and Conservation 28(5):1049-1073. https://doi.org/10.1007/s10531-019-01706-x

Lynch JD (2005) Discovery of the richest frog fauna in the world. An exploration of the forests to the North of Leticia. Revista de la Academia Colombiana de Ciencias Exactas, Físicas y Naturales 29: 581-588.

Maciel AO, Santana DJ, da Silva ET, Feio RN (2009) Amphibia, Gymnophiona, Caeciliidae, Siphonops hardyi Boulenger 1888: Distribution extension, new state record and notes on meristic data. Check List 5: 919-921. https://doi.org/10.15560/5.4.919

Maciel AO, Hoogmoed MS (2011) Taxonomy and distribution of caecilian amphibians (Gymnophiona) Of Brazilian Amazonia, with a key to their identification. Zootaxa 2984: 1-53. https://doi.org/10.11646/zootaxa.2984.1.1

Marciano-Jr E, Lantyer-Silva ASF, Solé M (2017) A new species of Phyllodytes Wagler 1830 (Anura, Hylidae) from the Atlantic Forest of southern Bahia, Brazil. Zootaxa 4238: 135142. https://doi.org/10.11646/zootaxa.4238.1.11

Mendes SL, Padovan MP (2000) A Estação Biológica de Santa Lúcia, Santa Teresa, Espírito Santo. Boletim Museu Biologia Mello Leitão 11/12: 7-34.

Mônico AT, Ferreira RB, Lauvers WD, Mattos RO, Clemente Carvalho RBG (2016) Itapotihyla langsdorffi (Perereca castanhola; Ocellated Treefrog). Head Abnormality. Herpetological Review 47: 278-279.

Olson DM, Dinerstein E, Wikramanayake ED, Burgess ND, Powell GVN, Underwood EC, D’amico JA, Itoua I, Strand HE, Morrison JC, Loucks CJ, Allnutt TF, Ricketts TH, Kura 
Y, Lamoreux JF, Wettengel WW, Hedao P, Kassem KR (2001) Terrestrial ecoregions of the world: a new map of life on Earth. BioScience 51: 933-938. https://doi.org/10.1641/00063568(2001)051[0933:TEOTWA]2.0.CO;2

Oommen VO, Measey GJ, Gower DJ, Wilkinson M (2000) Distribution and abundance of the caecilian Gegeneophis ramaswamii (Amphibia, Gymnophiona) in southern Kerala. Current Science 79: 1386-1389.

Passamani M, Mendes SL, Chiarello AG (2000) Non-volant mammals of the Estação Biológica de Santa Lúcia and adjacents areas of Santa Teresa, Espírito Santo, Brazil. Boletim do Museu de Biologia Mello Leitão 11/12: 201-214.

Pimenta BVS, Cruz CAG, Caramaschi U (2014) Taxonomic review of the species complex of Crossodactylus dispar Lutz 1925 (Anura, Hylodidae). Arquivos de Zoologia 45: 1-33. https://doi.org/10.11606/issn.2176-7793.v45i1p1-33

Pombal Jr JP, Gasparini JL (2006) A new Brachycephalus (Anura, Brachycephalidae) from the Atlantic Rainforest of Espírito Santo, southeastern Brazil. South American Journal Herpetology 1: 87-93. https://doi.org/10.2994/1808-9798(2006)1[87:ANBABF]2.0.CO;2

Ramos AD, Gasparini JL (2004) Anfíbios do Goiapaba-Açu. Gráfica Santo Antônio, Fundão, Brazil, 75 pp.

Ribeiro MC, Metzger JP, Martensen AC, Ponzoni FJ, Hirota MM (2009) The Brazilian Atlantic forest: how much is left, and how is the remaining forest distributed? Implications for conservation. Biological Conservation 142: 1141-1153. https://doi.org/10.1016/j.biocon.2009.02.021

Rizzini CT (1979) Tratado de Fitogeografia do Brasil: aspectos sociológicos e florísticos. Editora Hucitec Ltda \& Ed. Universidade de São Paulo, São Paulo, 374 pp.

Rödder D, Teixeira RL, Ferreira RB, Dantas RB, Pertel W, Guarniere GJ (2007) Anuran hotspots: the municipality of Santa Teresa, Espírito Santo, southeastern Brazil. Salamandra 43: 91-110.

Rodríguez LO, Duellman WE (1994) Guide to the frogs of the Iquitos region, Amazonian Peru. University of Kansas Natural History Museum Special Publication 22: 1-80.

Rossa-Feres D, Garey MV, Caramaschi U, Napoli MF, Nomura FA, Bispo A, Brasileiro CA, Thomé MT, Sawaya RJ, Conte CE, Cruz CAG, Nascimento LB, Gasparini JL, Almeida AP, Haddad CFB (2017) Anfíbios da Mata Atlântica: Lista de espécies, histórico dos estudos, biologia e conservação. In: Monteiro Filho EML, Conte CE (Eds) Revisões em Zoologia: Mata Atlântica, Curitiba: Editora UFPR, 237-314.

Scaramuzza CAM, Simóes LL, Rodrigues ST, Accacio GM, Hercowitz M, Rosa MR, Goulart W, Pinagé ER, Soares MS (2011) Visão da Biodiversidade da Ecorregião Serra do Mar: domínio biogeográfico Mata Atlântica. Brasília, WWF-Brasil, 167 pp.

Schloegel LM, Ferreira CM, James TY, Hipolito M, Longcore JE, Hyatt AD, Yabsley M, Martins AMCRPF, Mazzoni R, Davies AJ, Daszak P (2010) The North American bullfrog as a reservoir for the spread of Batrachochytrium dendrobatidis in Brazil. Animal Conservation 13: 53-61. https://doi.org/10.1111/j.1469-1795.2009.00307.x

Segalla MV, Caramaschi U, Cruz CAG, Grant T, Haddad CFB, Garcia PCA, Berneck BVM, Langone JA (2016) Brazilian Amphibians: list of species. Herpetologia Brasileira 5: 34-46. 
Silva ET, Ribeiro-Filho OP, Feio RN (2011) Predation of native anurans by invasive bullfrogs in southeastern Brazil: spatial variation and effect of microhabitat use by prey. South American Journal of Herpetology 6: 1-10. https://doi.org/10.2994/057.006.0101

Silva ET, Peixoto MAA, Leite FSF, Feio RN, Garcia PCA (2018) Anuran distribution in a highly diverse region of the Atlantic Forest: the Mantiqueira mountain range in southeastern Brazil. Herpetologica 74: 294-305. https://doi.org/10.1655/Herpetologica-D-17-00025.1

Simon JE (2000) Composição da avifauna da Estação Biológica de Santa Lúcia, Santa Teresa ES. Boletim do Museu de Biologia Mello Leitáo 11/12: 149-170.

Simon JE, Peres J (2012) Revisão da distribuição geográfica de Phyllodytes kautskyi Peixoto, Cruz 1988 (Amphibia, Anura, Hylidae). Boletim do Museu de Biologia Mello Leitão 29: 17-30.

SOS Mata Atlântica, Fundação INPE - Instituto Nacional De Pesquisas Espaciais (2013) Atlas dos Remanescentes Florestais da Mata Atlântica. Período 2013-2014. http://www.sosma. org.br/ [accessed on 11 February 2017]

Silvano DL, Pimenta BVS (2003) Diversidade de anfíbios na Mata Atlântica do Sul da Bahia. In: Prado PI, Landau EC, Moura RT, Pinto LPS, Fonseca GAB, Alger K (Eds) Corredor de Biodiversidade na Mata Atlântica do Sul da Bahia. IESB, CI, CABS, UFMG, UNICAMP, Ilhéus, CD-ROM.

Stuart S, Chanson J, Cox N, Young B, Rodrigues A, Fischman D, Waller R (2004) Status and trends of amphibian declines and extinctions worldwide. Science 306: 1783-1786. https:// doi.org/10.1126/science.1103538

Tabarelli M, Aguiar AV, Ribeiro MC, Metzger JP, Peres CA (2010) Prospects for biodiversity conservation in the Atlantic forest: lessons for aging human-modified landscapes. Biological Conservation 143: 2328-2340. https://doi.org/10.1016/j.biocon.2010.02.005

Thomaz LD, Monteiro R (1997) Composição florística da Mata Atlântica de encosta da Estação Biológica de Santa Lúcia, município de Santa Teresa, Espírito Santo. Boletim Museu Biologia Mello Leitão 7: 3-48.

Verdade VK, Rodrigues MT (2007) Taxonomic review of Allobates (Anura, Aromobatidae) from the Atlantic Forest, Brazil. Journal of Herpetology 41: 566-580. https://doi. org/10.1670/06-094.1

Verdade VK, Rodrigues MT, Pavan D (2009) Anfíbios anuros da Reserva Biológica de Paranapiacaba e entorno, pp. 579-604. In: Lopes IMS, Kirizawa M, Melo MRF (Eds) Patrimônio da Reserva Biológica do Alto da Serra de Paranapiacaba. A antiga Estação Biológica do Alto da Serra, Instituto de Botânica, São Paulo, 720 pp.

Verdade VK, Valdujo PH, Carnaval ACOQL, Schiesari C, Toledo LF, Mott T, Andrade G, Eterovick PC, Menin M, Pimenta BVS, Lisboa CS, Paula DC, Silvano D (2012) A leap further: the Brazilian Amphibian Conservation Action Plan. Alytes International Journal of Batrachology 29: 28-43.

Walker M, Gasparini JL, Haddad CFB (2016) A new polymorphic species of egg-brooding frog of the genus Fritziana from southeastern Brazil (Anura, Hemiphractidae). Salamandra 52: 221-229.

Weygoldt P (1986) Beobachtungen Zur Ökologie und biologie von fröschen an einem neotropischen bergbach. Zoologische Jahrbücher (Systematik) 113: 429-454. 
Weygoldt P (1989) Changes in the composition of mountain stream frog communities in the Atlantic mountains of Brazil: frogs as indicators of environmental deterioration? Studies on Neotropical Fauna and Environment 243: 249-255. https://doi. org/10.1080/01650528909360795

Wilkinson M, Nussbaum RA (1999) Evolutionary relationships of the lungless caecilian Atretochoana eiselti (Amphibia, Gymnophiona, Typhlonectidae). Zoological Journal of the Linnean Society 126: 191-223. https://doi.org/10.1111/j.1096-3642.1999.tb00153.x

Young BE, Stuart SN, Chanson JS, Cox NA, Boucher TM (2004) Disappearing jewels: The status of new world amphibians. Nature Serve, Arlington, VA, 53 pp.

Zocca CZ, Tonini JFR, Ferreira RB (2014) Uso do espaço por anuros em ambiente urbano de Santa Teresa, Espírito Santo. Boletim do Museu de Biologia Mello Leitão 35: 105-117.

\section{Appendix I}

\section{Vouchers of examined specimens}

Adelophryne glandulata (MBML 9560), Allobates capixaba (MZUSP 53559), Aplastodiscus cavicola (MBML 9620), Aplastodiscus aff. engenioi (MBML 7901), Aplastodiscus weygoldti (MBML 9540), Boana albomarginata (MBML 9610), Boana albopunctata (MBML 9673), Boana crepitans (MBML 9624), Boana faber (MBML 9576), Boana pardalis (MBML 9577), Boana semilineata (MBML 9554), Bokermannohyla caramaschii (MBML 9552), Brachycephalus alipioi (MNRJ 25405), Ceratophrys aurita (MBML 591), Chiasmocleis capixaba (MBML 2644), Chiasmocleis schubarti (MBML 9599), Crossodactylodes bokermanni (MBML 3984), Crossodactylodes izecksohni (MBML 768), Crossodactylus aff.gaudichaudii (MBML 15), Crossodactylus timbuhy (MBML 13), Cycloramphus fuliginosus (USNM 200441), Dendrophryniscus carvalhoi (MBML 8722), Dendropsophus berthalutzae (MBML 8589), Dendropsophus bipunctatus (MBML 2446), Dendropsophus branneri (MBML 9611), Dendropsophus bromeliaceus (MBML 7712), Dendropsophus decipiens (MBML 9590), Dendropsophus elegans (MBML 9543), Dendropsophus giesleri (MBML 8795), Dendropsophus haddadi (MBML 8775), Dendropsophus microps (MNRJ 30445), Dendropsophus minutus (MBML 9593), Dendropsophus ruschii (CFBH 37010), Dendropsophus seniculus (MBML 9591), Euparkerella tridactyla (MBML 7585), Fritziana aff. fissilis (MBML 46), Fritziana tonimi (MBML 8604), Gastrotheca albolineata (MBML 47), Gastrotheca megacephala (MBML 9672), Haddadus binotatus (MBML 9621), Hylodes cf. babax (USNM 222553), Hylodes lateristrigatus (MBML 9595), Ischnocnema abdita (MBML 1143), Ischnocnema colibri (MBML 10568-10572), Ischnocnema aff. guentheri (MBML 4534), Ischnocnema cf. nasuta (MBML 4667), Ischnocnema oea (MBML 8705), Ischnocnema aff. parva sp. 1 (MBML 9550), Ischnocnema verrucosa (MBML 9569), Itapotihyla langsdorffii (MBML 8585), Leptodactylus cupreus (MBML 6845), Leptodactylus fuscus (MBML 6003), Leptodactylus aff. latrans (MBML 2077), Leptodactylus aff. spixi (MBML 2439), Macrogenioglottus alipioi (MBML 93), Myersiella microps (MBML 9561), Ololygon arduous (MBML 
9657), Ololygon argyreornata (MBML 2828), Ololygon cf. flavoguttata (MBML 9649), Ololygon heyeri (MBML 8581), Ololygon kautskyi (MBML 9594), Phasmahyla exilis (MNRJ 4120), Phrynomedusa marginata (MNRJ 46881), Phyllodytes luteolus (MBML 6785), Phyllodytes aff. luteolus (MBML 9658), Phyllomedusa burmeisteri (MBML 9581), Physalaemus crombiei (MBML 9542), Physalaemus cuvieri (MBML 9579), Physalaemus maculiventris (MBML 9567), Physalaemus cf. olfersii (MBML 2803), Pipa aff. carvalhoi (MBML 4519), Pithecopus aff. rohdei (MBML 9580), Proceratophrys boiei (MBML 142), Proceratophrys laticeps (MBML 3905), Proceratophrys moehringi (MBML 6409), Proceratophrys paviotii (MBML 9585), Proceratophrys phyllostomus (MBML 325), Proceratophrys schirchi (MBML 9677), Rhinella crucifer (MBML 9575), Rhinella granulosa (MBML 2573), Rhinella diptycha (MBML 687), Scinax alter (MBML 9612), Scinax cuspidatus (MBML 3594), Scinax eurydice (MBML 1128), Scinax fuscovarius (MBML 7820), Scinax hayii (MBML 4707), Scinax aff. perereca (MBML 508), Scinax cf. $x$-signatus (MBML 4542), Siphonops annulatus (MBML 8586), Siphonops hardyi (MBML 8909), Thoropa aff. lutzi (MNRJ 1373), Thoropa miliaris (MBML 9571), Thoropa petropolitana (MZUSP 27725), Trachycephalus mesophaeus (MBML 8793), Trachycephalus nigromaculatus (MBML 9213), Vitreorana aff. eurygnatha (MBML 9678), Vitreorana uranoscopa (MBML 3725), Zachaenus carvalhoi (MNRJ 84116). 